\title{
Triethoxysilane with oligo(ethylene oxide) substituent as film forming additive for graphite anode ${ }^{*}$
}

\author{
Xue-ying QIN ${ }^{1,2}$, Jing-lun $\mathrm{WANG}^{1}$, Dao-ping TANG ${ }^{1}$, Yong-jin MAI ${ }^{1}$, Ling-zhi ZHANG ${ }^{\dagger 11}$ \\ $\left({ }^{1}\right.$ Key Laboratory of Renewable Energy, Guangzhou Institute of Energy Conversion, \\ Chinese Academy of Sciences, Guangzhou 510640, China) \\ $\left({ }^{2}\right.$ University of Chinese Academy of Sciences, Beijing 100039, China) \\ †E-mail: lzzhang@ms.giec.ac.cn
}

Received Jan. 15, 2013; Revision accepted Apr. 7, 2013; Crosschecked June 21, 2013

\begin{abstract}
TESM2) was synthesized and used as an electrolyte additive to improve the performances of lithium-ion batteries (LIBs). The electrochemical properties of the electrolyte $(1 \mathrm{~mol} / \mathrm{L}$ lithium hexafluorophosphate $\left(\mathrm{LiPF}_{6}\right)$ /ethylene carbonate $(\mathrm{EC})$ :diethylene carbonate $(\mathrm{DEC})$ :dimethyl carbonate $\left.(\mathrm{DMC}), 1: 1: 1\right)$ with different contents of TESM2 were characterized by ionic conductivity measurement, galvanostatic charge/discharge test of graphite/Li half cells, and electrochemical impedance spectroscopy. Both the cycling performances and C-rate capabilities of graphite/Li half cells were significantly improved with an optimized content of $15 \%$ TESM2 in the electrolyte. The graphite/Li half cell delivered a very high specific capacity of $370 \mathrm{mAh} / \mathrm{g}$ at $0.2 \mathrm{C}$ rate without any capacity loss for 60 cycles, and retained a capacity of $292 \mathrm{mAh} / \mathrm{g}$ at $2 \mathrm{C}$ rate. The solid electrolyte interphase (SEI) film on the surface of the graphite anode was investigated by scanning electron microscopy (SEM) and energy dispersive spectroscopy (EDS), indicating that TESM2 was effectively involved in the formation of SEI film on the surface of graphite.
\end{abstract}

Key words: Triethoxysilane, Electrolyte additive, Solid electrolyte interphase, Graphite anode, Lithium-ion batteries (LIBs) doi: $10.1631 /$ jzus.A1300026

Document code: A CLC number: O646

\section{Introduction}

Lithium-ion batteries (LIBs) have attracted great interest since the 1990s because of their unique properties such as high energy density, high voltage, and long cycling life. With the increasing demand for electric vehicles (EVs), much effort has been devoted to developing LIBs with long cycling life and high rate capacity (Hao et al., 2010; Li et al., 2011).

The electrolyte is an important component in LIBs and plays a key role in the electrochemical

\footnotetext{
${ }^{\ddagger}$ Corresponding author

* Project supported by the National Natural Science Foundation of China (No. 50973112), the Hundred Talents Program of the Chinese Academy of Sciences (CAS), the CAS-Guangdong Collaboration Program (No. 20108), the Science \& Technology Project of Guangzhou (No. 11A44061500), and the Amperex Technology Ltd. (China)

(C) Zhejiang University and Springer-Verlag Berlin Heidelberg 2013
}

performances of LIBs by forming a solid electrolyte interphase (SEI) film on the surface of a graphite anode during the initial charging/discharging process. The structure and components of the SEI film critically determine the stability of SEI film, and thus have great impact on the performance of LIBs (Zhang, 2006; Walkowiak et al., 2010). Therefore, a large number of additives have been reported to improve the interfacial properties of LIBs, such as reduction-type additives vinylene carbonate (VC) (Aurbach et al., 2002; 2004; Contestabile et al., 2003), vinyl ethylene carbonate (VEC) (Hu et al., 2004), reaction-type additives $\mathrm{CO}_{2}$ (Sakamoto et al., 2001), and lithium bis(oxalato) borate (LiBOB) (Xu et al., 2002).

Recently, organsilicon compounds have been reported as electrolyte solvents for LIBs because of their unique properties, such as nonflammability, 
biocompatibility, and thermal and electrochemical stability (Zhang et al., 2008). Some organosilicon compounds have already been developed as functional electrolyte additives such as film forming (Markovsky et al., 2004; Schroeder et al., 2006a; 2006b; Ryu et al., 2008; Xia et al., 2008; Song and Baek, 2009; Walkowiak et al., 2008; 2010; Li et al., 2011), and flame retardant (Zhang et al., 2009; Li et al., 2011) additives. To date, phenyl-functionalized silane (Xia et al., 2008; Li et al., 2011), polyetherfunctionalized disiloxanes (Walkowiak et al., 2008; 2010) and silicon tripodands (Schroeder et al., 2006a; 2006b) have been reported as additives in propylene carbonate (PC)-based electrolytes for graphite anodes to prevent exfoliation of the graphite.

In this work, we synthesized a triethoxysilane with oligo(ethylene oxide) substituent, \{3-[2-(2methoxyethoxy) ethoxy]-propyl $\}$ triethoxysilane (TESM2), and used it as a film forming additive in the electrolyte of $1 \mathrm{~mol} / \mathrm{L}$ lithium hexafluorophosphate $\left(\mathrm{LiPF}_{6}\right) /$ ethylene carbonate $(\mathrm{EC})$ :diethylene carbonate (DEC):dimethyl carbonate (DMC) $(1: 1: 1$, defined as LB303) for a graphite anode. The film forming capability was characterized by the electrochemical impedance spectroscopy (EIS), scanning electron microscopy (SEM), and energy dispersive spectroscopy (EDS) analyses. The electrochemical performances of the electrolytes with different contents of TESM2 were investigated in graphite/Li half cells.

\section{Experimental}

\subsection{Materials}

2-(2-methyloxyethoxy) ethanol (99\%), triethoxysilane (97\%), and allyl bromide (97\%) were obtained (Aladdin Co., China). The triethoxysilane was dried with molecular sieves before use. Potassium hydroxide $(\mathrm{KOH}, 85 \%)$ was purchased from the Guangzhou Maolin Co. (China). The platinum(IV) oxide $\left(\mathrm{PtO}_{2}\right.$, metal content $\left.84.65 \%\right)$ was purchased from the Shaanxi Kaida Chemical Engineering Co. (China). Graphite was obtained from the Amperex Technology Ltd. (China). The electrolyte of LB303 (with water content below $20 \mathrm{ppm}$ ) was commercially available from the Zhangjiagang Guotai-Huarong Co. (China).

\subsection{Apparatus}

${ }^{1} \mathrm{H}$ NMR (nuclear magnetic resonance) and ${ }^{13} \mathrm{C}$ NMR spectra were taken on a Bruker avence 600 spectrophotometer. Ionic conductivity of electrolytes (LB303) with different contents of TESM2 at room temperature were measured using a YSI 3200 conductivity instrument equipped with an YSI 3253 conductivity cell (conductivity meter DDS-310). The water content of electrolytes was measured by the Karl-Fischer coulometric moisture titrator (831 KF). All the cells were assembled under a dry argon atmosphere in glove box (Mikrouna, $\mathrm{H}_{2} \mathrm{O}$ and $\mathrm{O}_{2}<$ 1 ppm). The constant discharge and charge measurements of the graphite/Li cells were carried out with a multi-channel battery test system (Neware BTS-610) using 2032 coin cells at room temperature. The EIS was measured on an electrochemical analyzer (BAS-ZAHNER IM6) with $5 \mathrm{mV}$ AC amplitude applied in the frequency range of $0.01 \mathrm{~Hz}$ to $100 \mathrm{kHz}$. SEM and EDS were performed on the Hitachi S4800 to inspect the surface morphology of the graphite electrode. Before SEM and EDS, the cycled graphite electrode was washed three times with pure DEC followed by vacuum drying overnight at room temperature.

\subsection{Cell preparation and electrochemical measurements}

The graphite electrode was prepared by coating a mixture of graphite, carbon black, and poly(vinyledene) diflouride (PVDF) with weight ratio 90:5:5 on copper foil. The separator was purchased from Celgard Co. (Celgard 2400, USA). The electrolytes were prepared by adding $0,5 \%, 10 \%$, $15 \%$, and $20 \%$ TESM2 to the electrolyte of LB303. The cycling performance tests of the cells were operated at a constant current density of $0.2 \mathrm{C}$ (74.4 mA/g) under $0.005-3 \mathrm{~V}$. The C-rate performance tests of the cells were operated at a constant current density of $0.2 \mathrm{C}$ (10 cycles), $0.5 \mathrm{C}$ (10 cycles), 1C (10 cycles), 2C (10 cycles), 4C (10 cycles), and 0.2C (2 cycles) under $0.005-3 \mathrm{~V}$. All cell tests were conducted at a constant temperature of $25^{\circ} \mathrm{C}$.

\subsection{Synthesis of TESM2}

Firstly, allyl bromide (0.8 mol, $96.8 \mathrm{~g})$ was added to a mixture of $\mathrm{KOH}(0.8 \mathrm{~mol}, 44.8 \mathrm{~g})$ and 
2-(2-methyloxyethoxy) ethanol ( $0.7 \mathrm{~mol}, 84 \mathrm{~g})$ in a two-necked flask at $0{ }^{\circ} \mathrm{C}$, then the mixture was stirred at $55^{\circ} \mathrm{C}$ overnight. After completion of the reaction, the product was extracted with toluene/water (1:1), and dried with $\mathrm{MgSO}_{4}$. After pumping off the solvent, the allyl substituted oligo(ethylene oxide) ether was purified through repeated vacuum distillation in the presence of $\mathrm{CaH}_{2}$ to remove trace water, giving the purity of $99 \%$ characterized by gas chromatography. TESM2 was produced by hydrosilylation reaction of allyl substituted oligo(ethylene oxide) ether $(0.3 \mathrm{~mol}$, $48 \mathrm{~g})$ and triethoxysilane $(0.3 \mathrm{~mol}, 49.2 \mathrm{~g})$ with $1 \%$ (in weight) $\mathrm{PtO}_{2}$ as a catalyst in the condition of $80{ }^{\circ} \mathrm{C}$ for $12 \mathrm{~h}$. Upon reaction completion, TESM2 was purified by repeated vacuum distillation giving a yield of $80 \%$ with a purity of $99.6 \%$. The TESM 2 was dried over molecular sieves to ensure water content below 20 ppm prior to use for electrochemical tests. The chemical structure of TESM2 was confirmed by NMR: ${ }^{1} \mathrm{H}$ NMR $\left(600 \mathrm{MHz}, \mathrm{CDCl}_{3}\right): 0.62(\mathrm{~m}, 2 \mathrm{H})$, $1.21(\mathrm{t}, \mathrm{J}=7 \mathrm{~Hz}, 9 \mathrm{H}), 1.69(\mathrm{~m}, 2 \mathrm{H}), 3.38(\mathrm{~s}, 3 \mathrm{H}), 3.42$ (t, J=7 Hz, 2H), $3.54(\mathrm{~m}, 2 \mathrm{H}), 3.58(\mathrm{~m}, 2 \mathrm{H}), 3.63(\mathrm{~m}$, $4 \mathrm{H}), 3.80$ (q, J=7 Hz, 6H); ${ }^{13} \mathrm{C}$ NMR $(109 \mathrm{MHz}$, $\left.\mathrm{CDCl}_{3}\right): 6.41,18.25,22.87,58.30,58.97,69.97$, $70.51,70.63,71.93,73.60$.

\section{Results and discussion}

\subsection{Synthesis and characterization}

The TESM2 was synthesized through a hydrosilylation reaction of triethoxysilane with oligo(ethylene oxide) allyl methyl ether using $\mathrm{PtO}_{2}$ as a catalyst to yield $80 \%$ (Scheme 1) (Ghosh et al., 2005). Its chemical structure was confirmed by NMR (Section 2). The basic physical properties of TESM2 at room temperature were collected, showing a low viscosity $(3.26 \mathrm{mPa} \cdot \mathrm{s})$, moderate dielectric constant (4.88) and ionic conductivity $(1.14 \mathrm{mS} / \mathrm{cm}$ doped with $1 \mathrm{~mol} / \mathrm{L}$ LiTFSI).

The dependence of ionic conductivity of the electrolytes with different doping content of TESM2 is plotted in Fig. 1. The ionic conductivity of the electrolytes decreased with increasing TESM2 content, reducing from $9.4 \mathrm{mS} / \mathrm{cm}$ without additive to $7.4 \mathrm{mS} / \mathrm{cm}$ with $20 \%$ addition of TESM2 in the electrolyte of LB303.

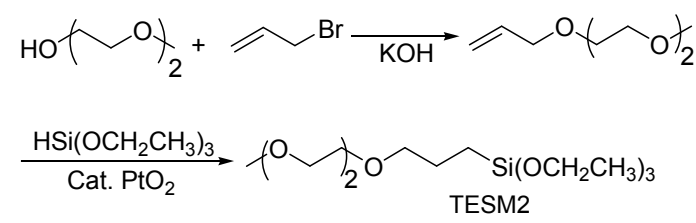

Scheme 1 Synthesis of silane-based compound TESM2

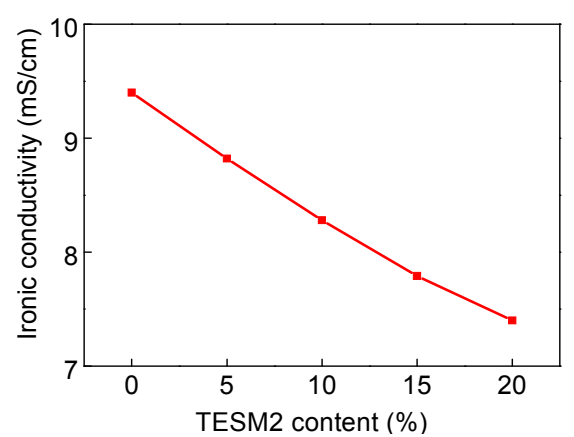

Fig. 1 Relationship between the ionic conductivity and doping content of TESM2 in LB303

\subsection{Cycle performance test}

Fig. 2 shows the cycling and C-rate performances of graphite/Li half cells with different contents of TESM2 in LB303 electrolyte. All the cycling performances of the cells were improved with $5 \%, 10 \%, 15 \%$, and $20 \%$ additions of TESM 2 without sacrificing the initial coulombic efficiency (the initial coulombic efficiencies of the cells with $0,5 \%, 10 \%$, $15 \%$, and $20 \%$ addition of TESM 2 are $82.0 \%, 79.5 \%$, $81.1 \%, 81.6 \%$, and $81.1 \%$, respectively), as compared with the control cell without additive (Fig. 2a). The cell with $20 \%$ addition exhibited the highest capacity of $370 \mathrm{mAh} / \mathrm{g}$ and showed no capacity loss after 60 cycles. Furthermore, the $\mathrm{C}$-rate performances of the cells (with charge/discharge rates of $1 \mathrm{C}, 2 \mathrm{C}$, and $4 \mathrm{C}$ ) were also improved with $5 \%, 10 \%$, and $15 \%$ additions of TESM2, but not with $20 \%$ addition (Fig. 2 b). The graphite/Li cell with $15 \%$ addition displayed the best $\mathrm{C}$-rate performance at $4 \mathrm{C}$ rate. Moreover, the higher doping content of TESM2 deteriorated the rate performances as seen in the case of $20 \%$ addition, probably due to its low ionic conductivity (Fig. 1).

\subsection{Impedance analysis}

The EIS and surface analysis of these graphite electrodes were carried out to investigate the underlying impact of the doping content of TESM2 on the cycling and C-rate performance. The impedance of graphite/Li cells with different contents of TESM2 
in LB303 after 4 cycles are shown in Fig. 3. In the Nyquist plots, the semicircles at high frequency are related to the SEI film resistance and the impedance of lithium ion transport through the interphase of electrolyte and electrode (Hao et al., 2010; Li et al., 2011). The SEI film resistances of the cells with $5 \%$, $10 \%$, and $15 \%$ additions were $14.0 \Omega, 15.0 \Omega$, and $15.5 \Omega$, respectively, as compared with higher values of $18.0 \Omega$ and $17.7 \Omega$ for the cell without and with $20 \%$ addition in the electrolyte, respectively. This decrease of the resistance with proper doping content implies a higher lithium ion migration rate and better kinetic characters, thus the C-rate performance of the cells was improved.
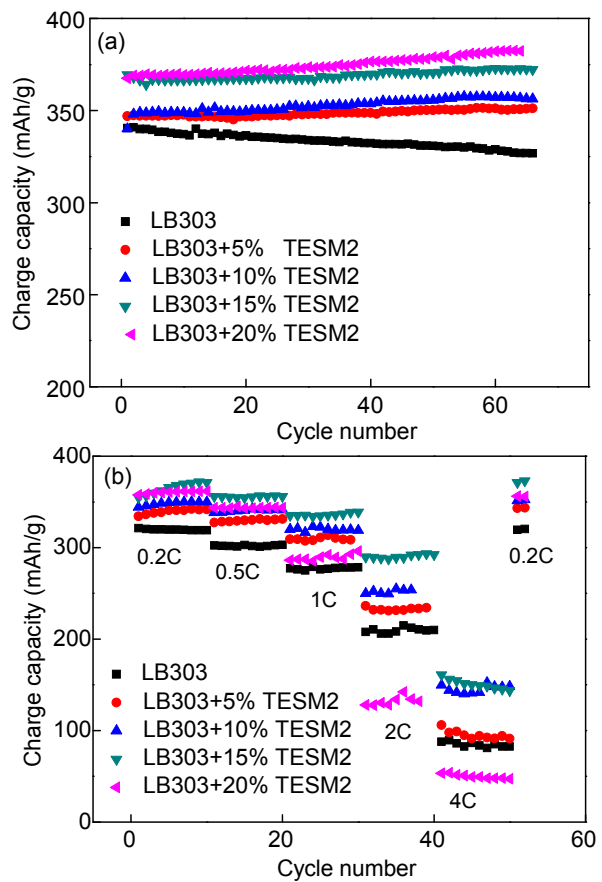

Fig. 2 Cycling performance at $0.2 \mathrm{C}$ rate (a) and $\mathrm{C}$-rate performance (b) of graphite/Li batteries with different doping contents of TESM2 in LB303

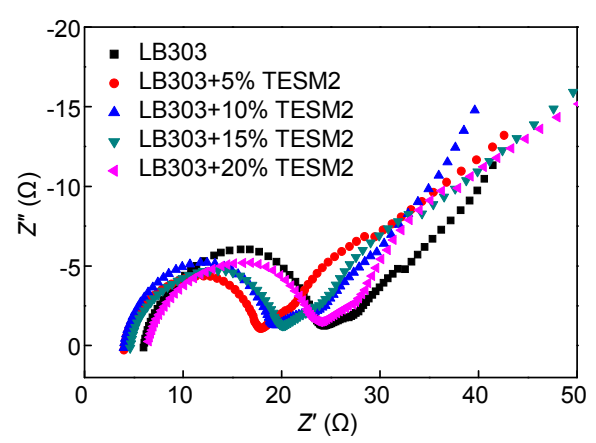

Fig. 3 Impedance of graphite/Li batteries with different doping contents of TESM2 added in LB303 after 4 cycles

\subsection{Analyses of graphite anode surface}

To further study the effect of TESM2 on the surface modification of graphite anode, SEM analysis was conducted to characterize the surface morphologies of graphite electrodes. For the cell without additive (Fig. 4a), there was more fluff-like and particle-like materials covered on the surface of graphite; while the surface of graphite with $10 \%$ additive became smoother and had large-sized particles (Fig. 4b).

(a)

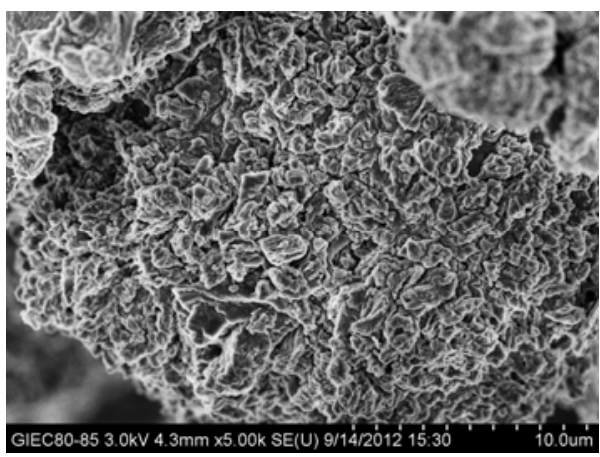

(b)

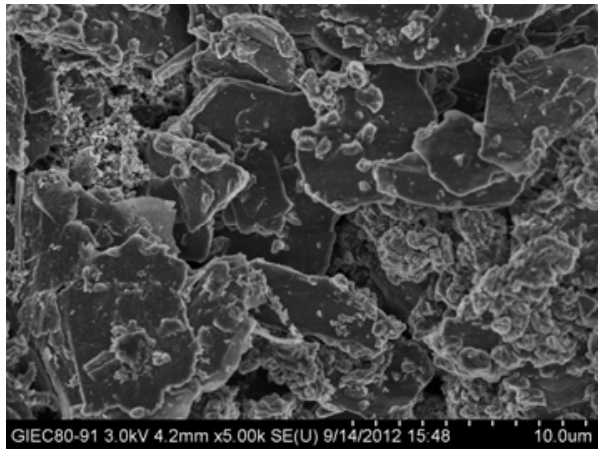

Fig. 4 SEM micrographs of graphite electrode without TESM2 (a) and with 10\% TESM2 after 3 cycles (b)

The surface film components of the graphite anode without and with 10\% TESM2 addition in LB303 were further studied by EDS (Fig. 5). A silicon peak at $1.8 \mathrm{keV}$ was detected for the sample of the latter cell, indicating that TESM2 was obviously involved in the formation of SEI film on graphite anode. No silicon signal was observed for the sample of the cell without additive. On the other hand, the increase of the carbon signal in the EDS spectrum of the sample with TESM2 addition further evidenced that TESM2 was involved in the formation of SEI film, as the carbon content relative to oxygen of TESM2 was higher than EC. Apparently, TESM2 participated in the formation of SEI film on graphite 
anode, and thus improved the cycling and C-rate performance.

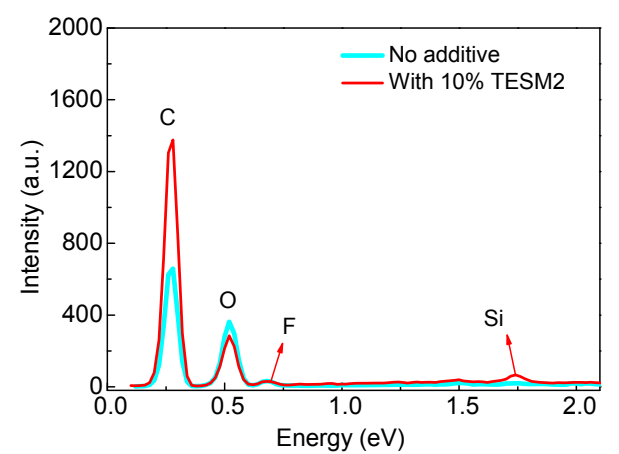

Fig. 5 EDS spectrum of graphite electrode without and with $10 \%$ TESM2 after 3 cycles

\section{Conclusions}

Oligo(ethylene oxide) functionalized triethoxysilane (TESM2) was synthesized as film forming additive for graphite anode in lithium-ion batteries. In an electrolyte of $1 \mathrm{~mol} / \mathrm{L} \mathrm{LiPF}_{6} / \mathrm{EC}: \mathrm{DEC}$ : DMC with an optimized content of triethoxysilane compound $(15 \%)$, both the cycling performances and C-rate capabilities of graphite/Li half cells are significantly improved. The graphite/Li half cells delivered a very high specific capacity of $370 \mathrm{mAh} / \mathrm{g}$ at $0.2 \mathrm{C}$ without any capacity loss for 60 cycles, and retained a capacity of $292 \mathrm{mAh} / \mathrm{g}$ at 2C rate. EIS, SEM, and EDS analyses disclosed that the TESM2 participated in the formation of stable and effective SEI film on the surface of graphite electrode, thus improved the performance of graphite half cells. Therefore, triethoxysilane compound with oligo(ethylene oxide) substituent is a promising additive candidate to be used for improving the performances of LIBs.

\section{References}

Aurbach, D., Gamolsky, K., Markovsky, B., Gofer, Y., Schmidt, M., Heider, U., 2002. On the use of vinylene carbonate (VC) electrolyte solutions for Li-ion as an additive to batteries. Electrochimica Acta, 47(9):1423-1439. [doi:10. 1016/S0013-4686(01)00858-1]

Aurbach, D., Gnanaraj, J.S., Geissler, W., Schmidt, M., 2004. Vinylene carbonate and $\mathrm{Li}$ salicylatoborate as additives in $\mathrm{LiPF}_{3}\left(\mathrm{CF}_{2} \mathrm{CF}_{3}\right)_{3}$ solutions for rechargeable Li-ion batteries. Journal of the Electrochemical Society, 151(1): A23-A30. [doi:10.1149/1.1631820]

Contestabile, M., Morselli, M., Paraventi, R., Neat, R.J., 2003. A comparative study on the effect of electrolyte/additives on the performance of ICP383562Li-ion polymer (soft-pack) cells. Journal of Power Sources, 119-121(SI): 943-947. [doi:10.1016/S0378-7753(03)00292-1]

Ghosh, B.D., Lott, K.F., Ritchie, J.E., 2005. Conductivity dependence of PEG content in an anhydrous proton conducting sol-gel electrolyte. Chemistry of Materials, 17(3):661-669. [doi:10.1021/cm0486969]

Hao, X., Liu, P., Zhang, Z., Lai, Y., Wang, X., Li, J., Liu, Y., 2010. Tetraethylammonium tetrafluoroborate as additive to improve the performance of $\mathrm{LiFePO}_{4} /$ artificial graphite cells. Electrochemical and Solid-State Letters, 13(8): A118-A120. [doi:10.1149/1.3439642]

Hu, Y.S., Kong, W.H., Wang, Z.X., Li, H., Huang, X., Chen, L.Q., 2004. Effect of morphology and current density on the electrochemical behavior of graphite electrodes in PC-based electrolyte containing VEC additive. Electrochemical and Solid-State Letters, 7(11):A442-A446. [doi:10.1149/1.1807532]

Li, L.L., Wang, B., Liu, L.L., Wu, Y.P., van Ree, T., Thavhiwa, K.A., 2011. Methyl phenyl bis-methoxydiethoxysilane as bi-functional additive to propylene carbonate-based electrolyte for lithium ion batteries. Electrochimica Acta, 56(13):4858-4864. [doi:10.1016/j.electacta.2011.02.117]

Markovsky, B., Nimberger, A., Talyosef, Y., Rodkin, A., Belostotskii, A.M., Salitra, G., Aurbach, D., Kim, H.J., 2004. On the influence of additives in electrolyte solutions on the electrochemical behavior of carbon/ $\mathrm{LiCoO}_{2}$ cells at elevated temperature. Journal of Power Sources, 136(2):296-302. [doi:10.1016/j.powsour.2004. 04.017]

Ryu, Y.G., Lee, S.S., Mah, S.K., Lee, D.J., Kwon, K., Hwang, S.S., Doo, S.G., 2008. Electrochemical behaviors of silicon electrode in lithium salt solution containing alkoxy silane additives. Journal of the Electrochemical Society, 155(8):A583-A589. [doi:10.1149/1.2940310]

Sakamoto, J.S., Wudlb, F., Dunn, B., 2001. Passivating lithium electrodes with trimethylsilylacetylene. Solid State Ionics, 144(3-4):295-299. [doi:10.1016/S0167-2738(01)00902$\mathrm{X}]$

Schroeder, G., Gierczyk, B., Waszak, D., Kopczyk, M., Walkowiak, M., 2006a. Vinyl tris-2-methoxyethoxy silane-A new class of film-forming electrolyte components for $\mathrm{Li}$-ion cells with graphite anodes. Electrochemistry Communications, 8(4):523-527. [doi:10. 1016/j.elecom. 2006.01.021]

Schroeder, G., Gierczyk, B., Waszak, D., Walkowiak, M., 2006b. Impact of ethyl tris-2-methoxyethoxy silane on the passivation of graphite electrode in $\mathrm{Li}$-ion cells with PC-based electrolyte. Electrochemistry Communications, 8(10):1583-1587. [doi:10.1016/j.elecom.2006.07.030]

Song, S.W., Baek, S.W., 2009. Silane-derived SEI stabilization on thin-film electrodes of nanocrystalline Si for lithium batteries. Electrochemical and Solid-State Letters, 12(2): A23-A27. [doi:10.1149/1.3028216]

Walkowiak, M., Waszak, D., Schroeder, G., Gierczyk, B., 2008. Polyether-functionalized disiloxanes as new film-forming electrolyte additive for $\mathrm{Li}$-ion cells with graphitic anodes. 
Electrochemistry Communications, 10(11):1676-1679. [doi:10.1016/j.elecom.2008.08.036]

Walkowiak, M., Waszak, D., Schroeder, G., Gierczyk, B., 2010. Enhanced graphite passivation in Li-ion battery electrolytes containing disiloxane-type additive/co-solvent. Journal of Solid State Electrochemistry, 14(12):2213-2218. [doi:10.1007/s10008-008-0710-4]

Xia, Q., Wang, B., Wu, Y.P., Luo, H.J., Zhao, S.Y., van Ree, T., 2008. Phenyl tris-2-methoxydiethoxy silane as an additive to PC-based electrolytes for lithium-ion batteries. Journal of Power Sources, 180(1):602-606. [doi:10. 1016/j.jpowsour.2008.01.039]

Xu, K., Zhang, S.S., Jow, T.R., Xu, W., Angell, C.A., 2002. LiBOB as salt for lithium-ion batteries-A possible solution for high temperature operation. Electrochemical and Solid-State Letters, 5(1):A26-A29. [doi:10.1149/1. 1426042]
Zhang, S.S., 2006. A review on electrolyte additives for lithium-ion batteries. Journal of Power Sources, 162(2): 1379-1394. [doi:10.1016/j.jpowsour.2006.07.074]

Zhang, H.P., Xia, Q., Wang, B., Yang, L.C., Wu, Y.P., Sun, D.L., Gan, C.L., Luo, H.J., Bebeda, A.W., van Ree, T., 2009. Vinyl-tris-(methoxydiethoxy)silane as an effective and ecofriendly flame retardant for electrolytes in lithium ion batteries. Electrochemistry Communications, 11(3): 526-529. [doi:10.1016/j.elecom.2008.11.050]

Zhang, L.Z., Zhang, Z.C., Harring, S., Straughan, M., Butorac, R., Chen, Z.H., Lyons, L., Amine, K., West, R., 2008. Highly conductive trimethylsilyl oligo(ethylene oxide) electrolytes for energy storage applications. Journal of Materials Chemistry, 18(31):3713-3717. [doi:10.1039/ b806290k]

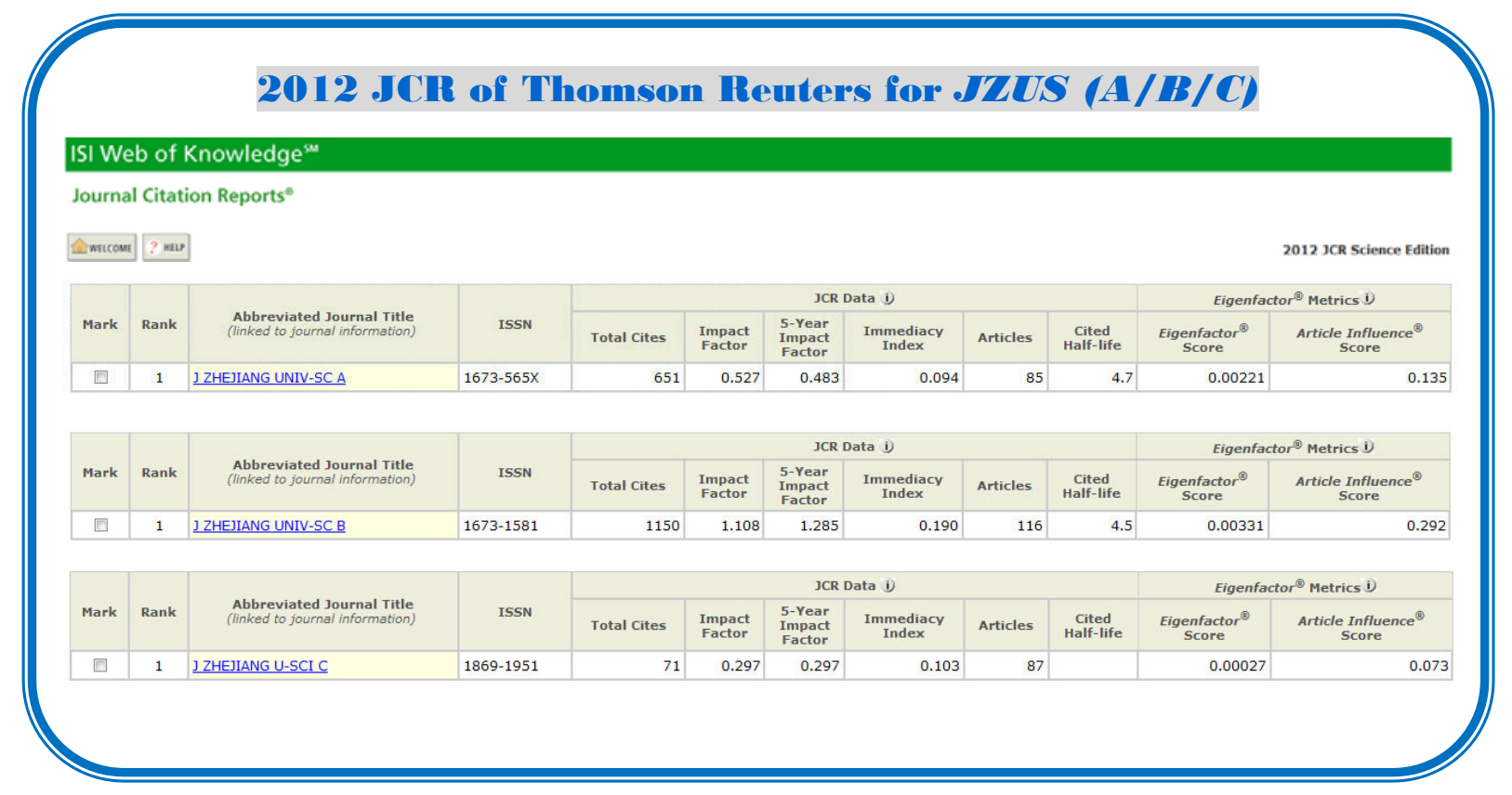

